\title{
A FACE RECOGNITION USING LINEAR-DiAgONAL BINARY GRAPH PATTERN FEATURE EXTRACTION METHOD
}

\author{
Satyendra Rajput and Dr. Joyti Bharti \\ Department of Computer Science and Engineering, MANIT Bhopal, Madhya Pradesh, \\ India
}

\begin{abstract}
Face recognition is one the most interesting topic in the field in computer vision and image processing. Face recognition is a processing system that recognizes and identifies individuals human by their faces. Automatic face recognition is powerful way to provide, authorized access to control their system. Face recognition has many challenging problems (like face pose, face expression variation, illumination variation, face orientation and noise) in the field of image analysis and computer vision. This method is work on feature extraction part of face recognition. New way to extract face feature using $L D-B G P$ code operator it is like LGS and LBP feature extraction operator. In our LD-BGP-code operator work in two direction first linear then diagonal. In both direction, its create eight digits code to every pixel of image. Means of these two directional are taken so that is cover all neighbor of center pixel. First linear direction, only horizontal and vertical pixel are taken. Second diagonal direction only diagonal pixels taken. In matching phase, we use Euclidean distance to match a face image. We perform the Linear and diagonal directional operator method on face database ORL. We get accuracy $95.3 \%$. LD-BGP method also works on different type image like illuminated and expression variation image.
\end{abstract}

\section{KEYWORDS}

face recognition, text based local descriptor, biometric, pattern recognition and LD-BGP.

\section{INTRODUCTION}

Face recognition is one of the field of biometrics and computer vision system. Using digital face image we analysis different individual person and recognized every individual with their corresponding digital image. It has a wide range of applications in information security, image retrieval, and access control and law enforcement surveillance. The area of face recognition has achieved a significant research progress. This is due to the current systems performing well under relatively controlled environments but tend to suffer when variations in different factors, such as aging, illumination, facial expressions and pose, are present. Thus the main goal of the ongoing research is to increase the performance and robustness of the system against various factors. Mainly face recognition system task work in three phase figure 1. 


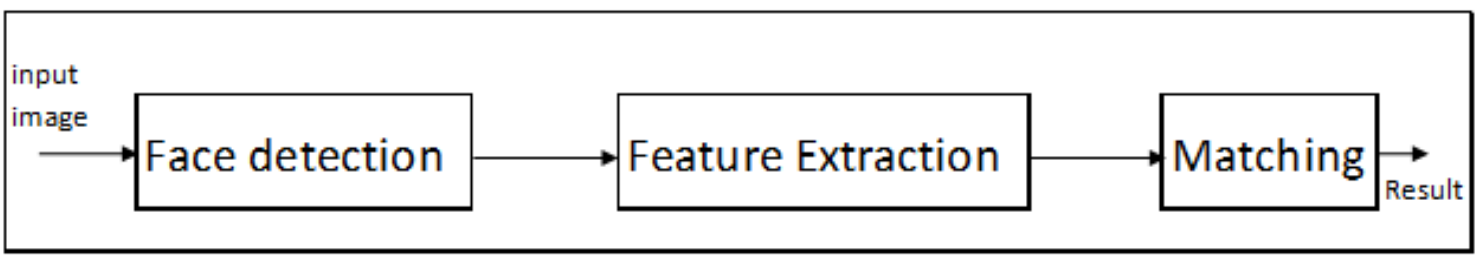

Figure 1.Phases of face recognition system

Various approaches has been proposed in face recognition field. Eigen face was proposed by Mathrew and Alex they use eigen value and eigen vector concept in face recognition problem. [5]Other approach like LDA isused for face recognition system it is also known as fisher face. [6] These two method is dimension reduction techniques that are reduce higher dimension into lower dimension. Template matching method is also used in face recognition problem in which template of facial feature is generate and do a template matching of each person image[7]. Neural network is also in face recognition problem two basic neural network first feed forward second back propagation neural network is used. [8, 9].

\section{General DifFiCUlties}

There are two major factor that effect the face recognition problem. 1) Intrinsic factors 2) extrinsic factors, [2] Intrinsic factors are due to the physical nature of the face and are independent of the observer. These factors can be further divided into two classes: intrapersonal and interpersonal. Intrapersonal factors are responsible for varying the facial appearance of the same person, some examples being age, facial expression and facial paraphernalia (facial hair, glasses, cosmetics, etc.). Interpersonal factors, however, are responsible for the differences in the facial appearance of different people, some examples being ethnicity and gender.[2] Other extrinsic factors cause the appearance of the face to alter via the interaction of light with the face and the observer. These factors include illumination, Expression variation, face occlusion, tilted face pose, scale and imaging parameters (e.g., resolution, focus, imaging, noise, etc.)
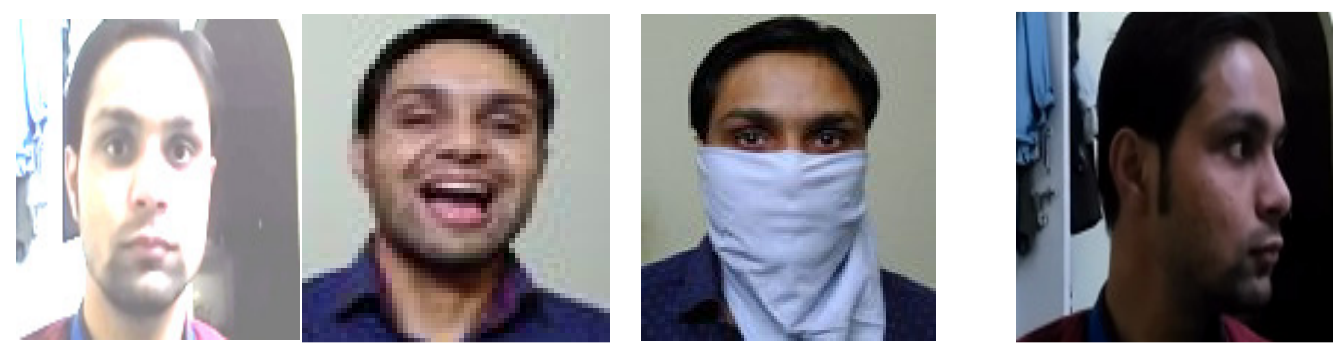

Figure 2. General difficulties i) illumination variation ii) expression change iii) occluded face iv) titled face

\section{FAcial Feature}

Every facial is almost unique to identify the each person. For which feature of face is extracted by different method. Research also has proposed different method for face feature extraction. Basically two type of facial feature extraction method 1) local feature 2) holistic feature, In local feature method we work on local feature of face image and holistic method hole image is consider as feature to uniquely recognized individual. 


\subsection{Local Binary Pattern}

Ojala et al. propsed the LBP operator which was originally designed for texture description and analysis.[12] The operator assigns a label to every pixel of an image by thresholding the $3 \times 3$ neighborhood of each pixel with the center pixel value and considering the result as a binary number. Then, the histogram of the labels can be used as a texture descriptor. [4]

To be able to deal with textures at different scales, the LBP operator was later extended to use neighborhoods of different sizes.
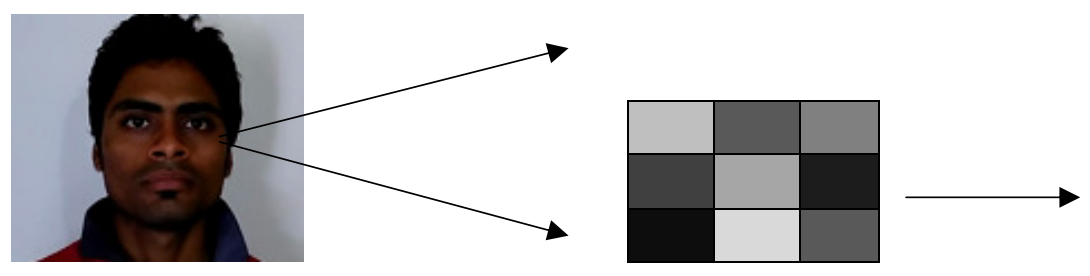

A) Image

\begin{tabular}{|c|c|c|}
\hline 52 & 4 & 9 \\
\hline 65 & 61 & 63 \\
\hline 67 & 33 & 85 \\
\hline
\end{tabular}

C) Grey values

B) One pixel

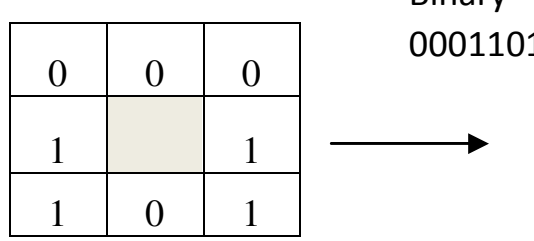

D) binary values

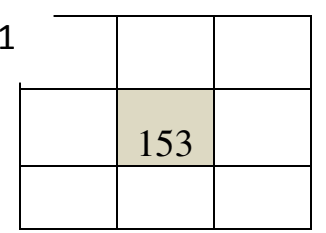

E) LBP values

Figure 3.Basic LBP operator

\subsection{Local Graph Structure}

It based on graph theory concept which is proposed by Eimad E.A. Abusham. LGS work on six neighboring pixel, it choose a target threshold pixel $\mathrm{C}$, then it starts by moving anti clockwise direction at the left side of the target $\mathrm{C}$ pixel, If the neighboring pixel has a higher gray value than that compared with the target pixel or it has same gray value, then it will assign 1 binary value on the edge which is connecting the two vertices, otherwise it will assign a 0 binary value. After finishing on the left side of graph we interlude for moment at the target pixel $\mathrm{C}$ and then it move in horizontal way to the right side of graph region in clockwise direction and it apply the similar process till the $\mathrm{C}$ target point is achieved. [1]

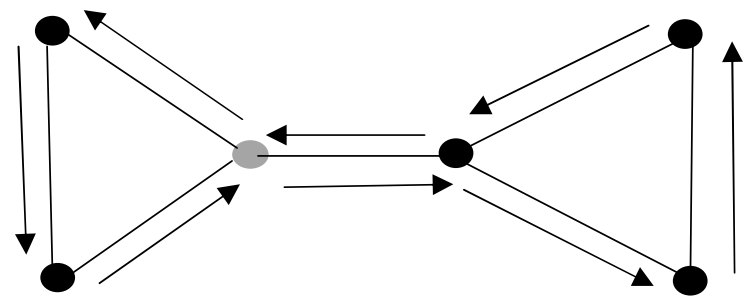

Figure 4. Local Graph structure operator 


\subsection{Symmetric Local Graph Structure}

SLGS is an extended version of LGS, it is proposed by Mohd Fikri Azli Abdullah in 2014. [3] This techniques is more balanced one as compared to LGS because it is symmetric to both side of the central pixel. Each pixel is related with six neighboring pixel in a radius of one and two. This techniques doesn't contain any redundant information as left side and right side of center pixel have a similar weightage. Figure 3, show the relationship of the neighbor pixels and the figure, shows SLGS operator.[3]

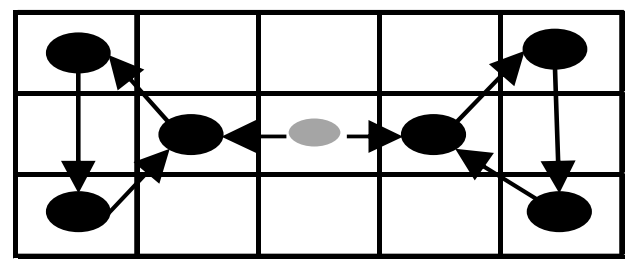

Figure 5. Relationship of neighbor pixel with center pixel

\begin{tabular}{|r|r|r|r|r|}
\hline 40 & 52 & 3 & 8 & 15 \\
\hline 61 & 45 & 60 & 62 & 58 \\
\hline 55 & 51 & 32 & 84 & 85 \\
\hline
\end{tabular}

Threshold

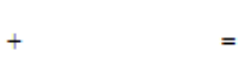

Binary001

01010
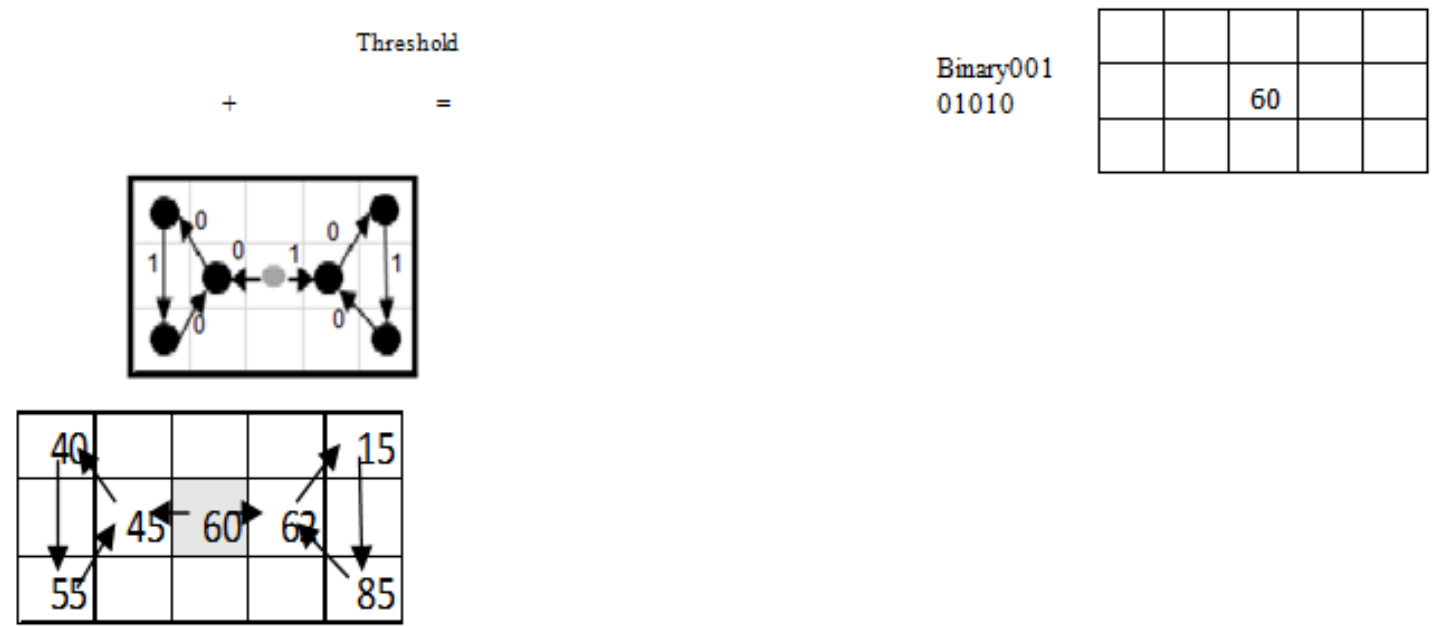

Figure 6. The SLGS Operator

SLGS method is very easy and simple. But majority of neighboring relationship lies only on horizontal pixels along left side and to the right side of the image. There is no focus on vertical pixels neighbor relationship.

Among three local feature techniques SLGS gives more accurate result in face recognition system. Comparison of these techniques is given in the table below: 
Table 1.Summary of different face recognition techniques

\begin{tabular}{|l|c|c|c|c|}
\hline Parameter & $\begin{array}{c}\text { Neighbor } \\
\text { pixels }\end{array}$ & $\begin{array}{c}\text { Radius of } \\
\text { neighbors }\end{array}$ & Based On & References \\
\hline LBP & 8 pixels & 1 (General case) & $\begin{array}{c}\text { Neighbor } \\
\text { connectivity }\end{array}$ & {$[4]$} \\
\hline LGS & 5 pixels & $\begin{array}{c}1 \text { left side, 2right } \\
\text { side }\end{array}$ & $\begin{array}{c}\text { Graph } \\
\text { structure }\end{array}$ & {$[3]$} \\
\hline SLGS & 6 pixels & 2 both side & $\begin{array}{c}\text { Symmetric } \\
\text { Graph }\end{array}$ & {$[1]$} \\
\hline
\end{tabular}

\subsection{LD-BGP}

In orl face database image size is $92 \mathrm{X} 112$ pixels values. Input image is grey image which is divided in $4 \mathrm{X} 4$ part means 16 equal part each part of image is size of $23 \mathrm{X} 28$. These subimage is called block of image. In each sub image total 456 pixel values are coded into LD-BGP-Code operator. So total 456 LD-BGP-Code are generated. Per image total is 16 X $456(16$ X 19 X 24) $=$ 7296 pixel values.

\begin{tabular}{|l|l|l|l|}
\hline $\begin{array}{l}\text { Block of } \\
\text { Image 1 } \\
\text { 23 X 28) }\end{array}$ & $\begin{array}{l}\text { Block of } \\
\text { Image 23 X 28) }\end{array}$ & $\begin{array}{l}\text { Block of } \\
\text { Image 3 } \\
\text { (23 X 28) }\end{array}$ & $\begin{array}{l}\text { Block of } \\
\text { Image 4 } \\
\text { (23 X 28) }\end{array}$ \\
$\begin{array}{l}\text { Block of } \\
\text { Image 5 }\end{array}$ & $\begin{array}{l}\text { Block of } \\
\text { Image 6 }\end{array}$ & $\begin{array}{l}\text { Block of } \\
\text { Image 7 }\end{array}$ & $\begin{array}{l}\text { Block of } \\
\text { Image 8 }\end{array}$ \\
\hline $\begin{array}{l}\text { Block of } \\
\text { Image 9 }\end{array}$ & $\begin{array}{l}\text { Block of } \\
\text { Image 10 }\end{array}$ & $\begin{array}{l}\text { Block of } \\
\text { Image 11 }\end{array}$ & $\begin{array}{l}\text { Block of } \\
\text { Image 12 }\end{array}$ \\
$\begin{array}{l}\text { Block of } \\
\text { Image 13 } \\
\text { (23 X 28) }\end{array}$ & $\begin{array}{l}\text { Block of } \\
\text { Image 14 X 28) }\end{array}$ & $\begin{array}{l}\text { Block of } \\
\text { Image 15 } \\
\text { (23 X 28) }\end{array}$ & $\begin{array}{l}\text { Block of } \\
\text { Image 16 } \\
\text { (23 X 28) }\end{array}$ \\
\hline
\end{tabular}

Figure 7. Blocks of image

In each blocks we apply Linear and Diagonal pattern method for feature extraction. Each direction pattern generates 8 bit code corresponding to every pixel. 


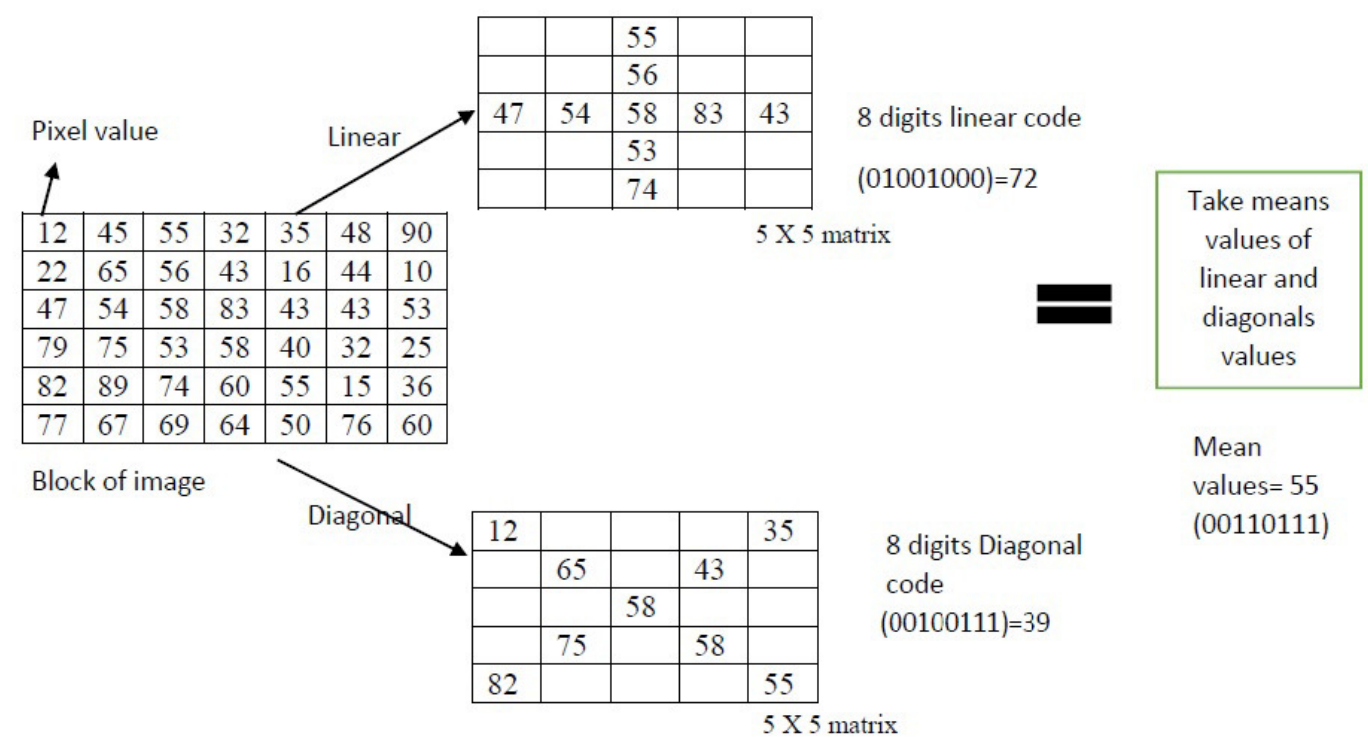

Figure 8. LD-BGP code operator

\subsubsection{Linear Directional Binary Pattern}

We take 5 X 5 matrix of image block. Then linear code is extraction corresponding to directional matrix shown below. We also assign a weight in directional matrix. Using weight we get eight digits code to center pixel value.

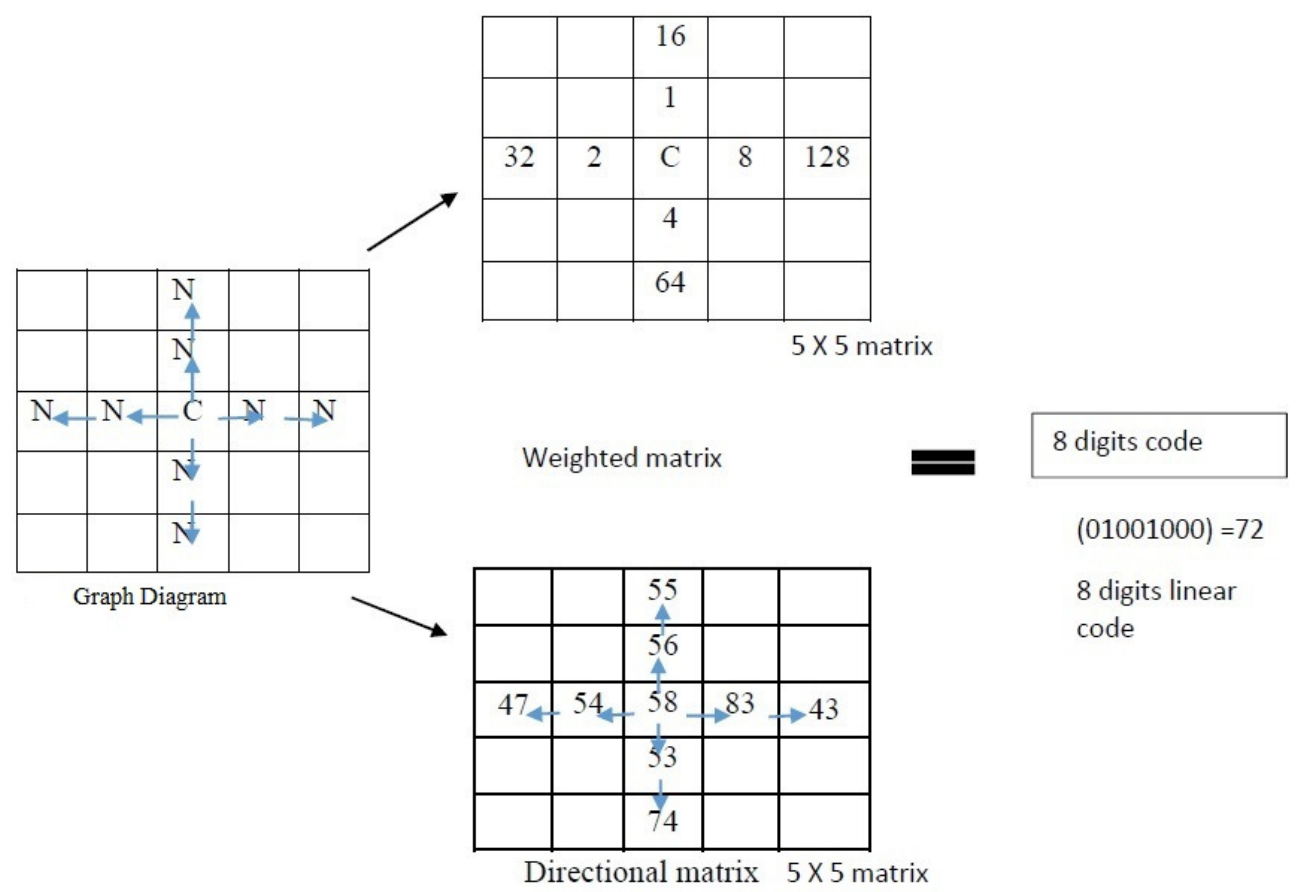

Figure 9.Linear direction binary pattern 
Where, $\mathrm{C}=$ center pixel values, $\mathrm{N}=$ neighbor of center pixel

Above figure shown directional matrix of $5 \times 5$ and weighted matrix that is used in linear LDBGP-Code extraction. In linear we take all linear neighbor that are left, right, above and below with radius of two. Total eight neighbors is used in linear LD-BGP-Code extraction method.

Linear LD-BGP-Code in equation (4.3)......

$\begin{aligned} P(c)= & (f(x-2, y)-f(c)),(f(x-1, y)-f(c)),(f(x, y+1)-f(c)),(f(x, y+2)-f(c)), \\ & (f(x, y-1)-f(c)),(f(x, y-2)-f(c)),(f(x+1, y)-f(c)),(f(x+2, y)-f(c))\}\end{aligned}$ $\ldots \ldots \ldots \ldots$ Equation $(1)$

Equation (1) shows general process of LD-BGP-Code extraction of center values.

\subsubsection{DIAGONAL DIRECTION BINARY PATTERN}

We take 5 X 5 matrix of image block. Then diagonal code is extraction corresponding to directional matrix shown below. We also assign a weight in directional matrix. Using weight we get eight digits code to center pixel value.

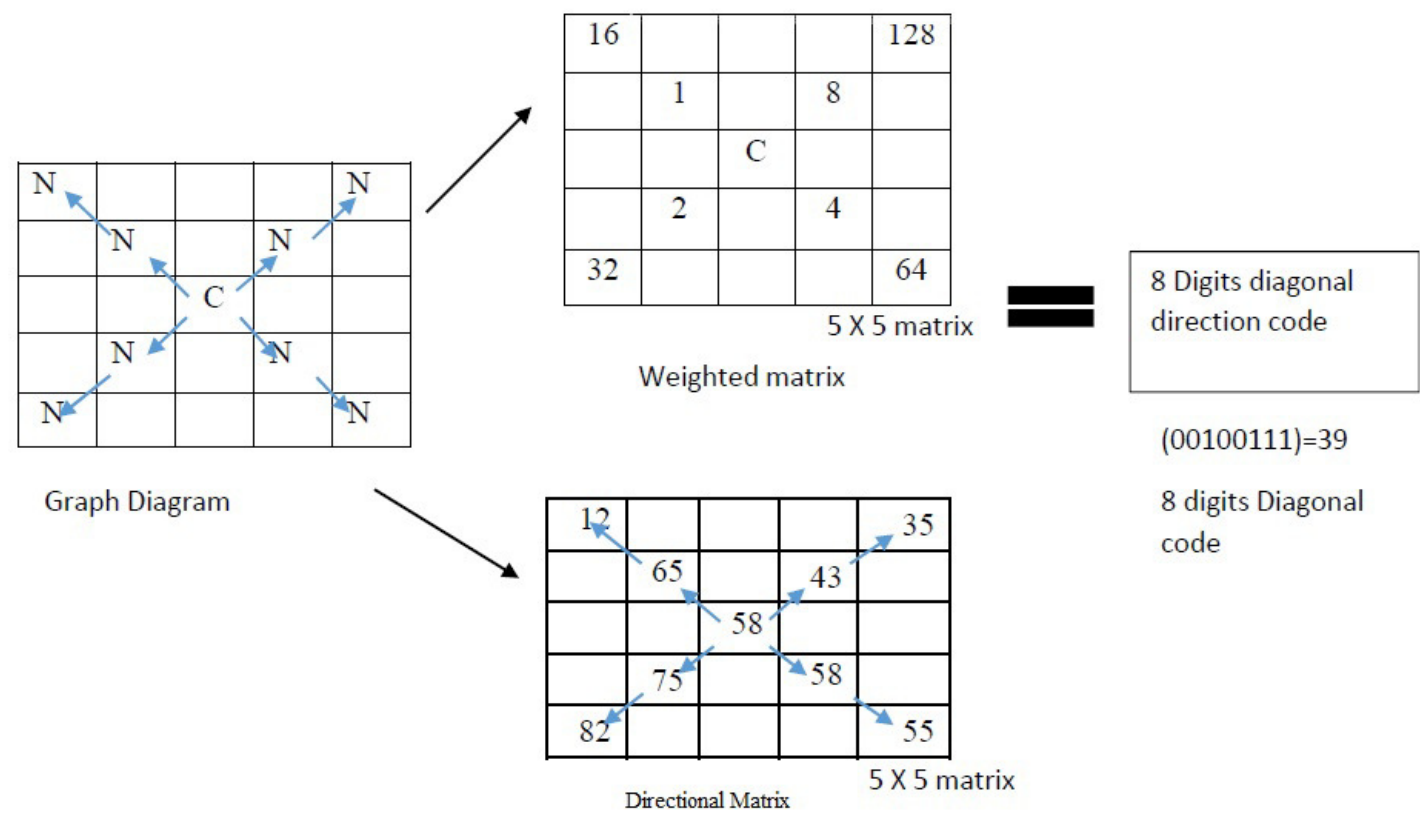

Figure 10. Diagonal direction LD-BGP-Code

Where, $\mathrm{C}=$ center pixel values, $\mathrm{N}=$ neighbor of center pixel

Figure 10 shown directional matrix of $5 \times 5$ and weighted matrix that is used in diagonal LDBGP-Code extraction. In linear we take all diagonal neighbor that are left corner above, right corner above, below left corner and below right corner with radius of two. Total eight neighbors is used in diagonal LD-BGP-Code extraction method. 
Diagonal LD-BGP-Code equation ......

$P(c)=\{((f(x-2, y-2)-f(c)),(f(x-1, y-1)-f(c)),(f(x-1, y+1)-f(c)),(f(x-2, y+2)-f(c))$, $(f(x+1, y-1)-f(c)),(f(x+2, y-2)-f(c)),(f(x+1, y+1)-f(c)),(f(x+2, y+2)-f(c))\} \ldots$ Equation $(2)$

\section{EXPERIMENTAL DESIGN}

Freely available orl face database (also known as AT \& T) is use to test performance of LD-BGP. [] In this database there are40 distinct person with ten different images of each person. For some person, the images were taken at different times, varying the lighting, facial expressions (open / closed eyes, smiling / not smiling) and facial details (glasses / no glasses). All the images were taken against a dark homogeneous background with the subjects in an upright, frontal position (with tolerance for some side movement).

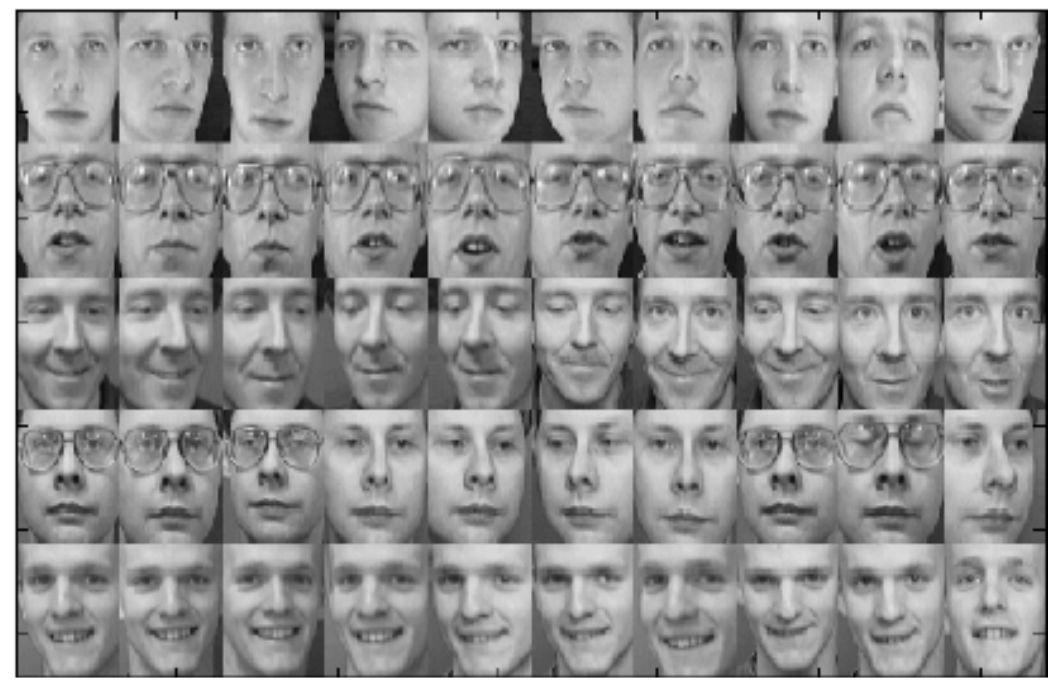

Figure 11: Sample image of ORL face

\section{RESULT}

Experiment is conducted to compare the effectiveness of distance measures which are Euclidean distance. The experiment used LDBGP for feature extraction algorithm on the ORL(AT\&T) database.

Table 1show accuracy on nearest neighbor with Euclidean distance of fine $\mathrm{K}$, medium $\mathrm{K}$ and weighted K nearest neighbor classifier. We get Overall maximum accuracy 95.30\%.

Table 2. Accuracy on ORL database

\begin{tabular}{|l|l|}
\hline Classifier & Accuracy \\
\hline Fine K-Nearest Neighbor & 93.30 \\
\hline Medium K-nearest Neighbor & 95.30 \\
\hline Weighted K-nearest Neighbor & 95.30 \\
\hline & \\
\hline Overall Maximum Accuracy & 95.3 \\
\hline
\end{tabular}


Table 3.Show accuracy of LD-BGP method using distance measure with different number of training files.

Table 3. Accuracy with different training files percentage.

\begin{tabular}{|l|l|}
\hline Training files \% & Accuracy \% (Ed) \\
\hline 10 & 89.58 \\
\hline 20 & 89.86 \\
\hline 30 & 90.28 \\
\hline 40 & 91.66 \\
\hline 50 & 92.18 \\
\hline 60 & 93.60 \\
\hline 70 & 94.40 \\
\hline 80 & 95.10 \\
\hline 90 & 95.30 \\
\hline
\end{tabular}

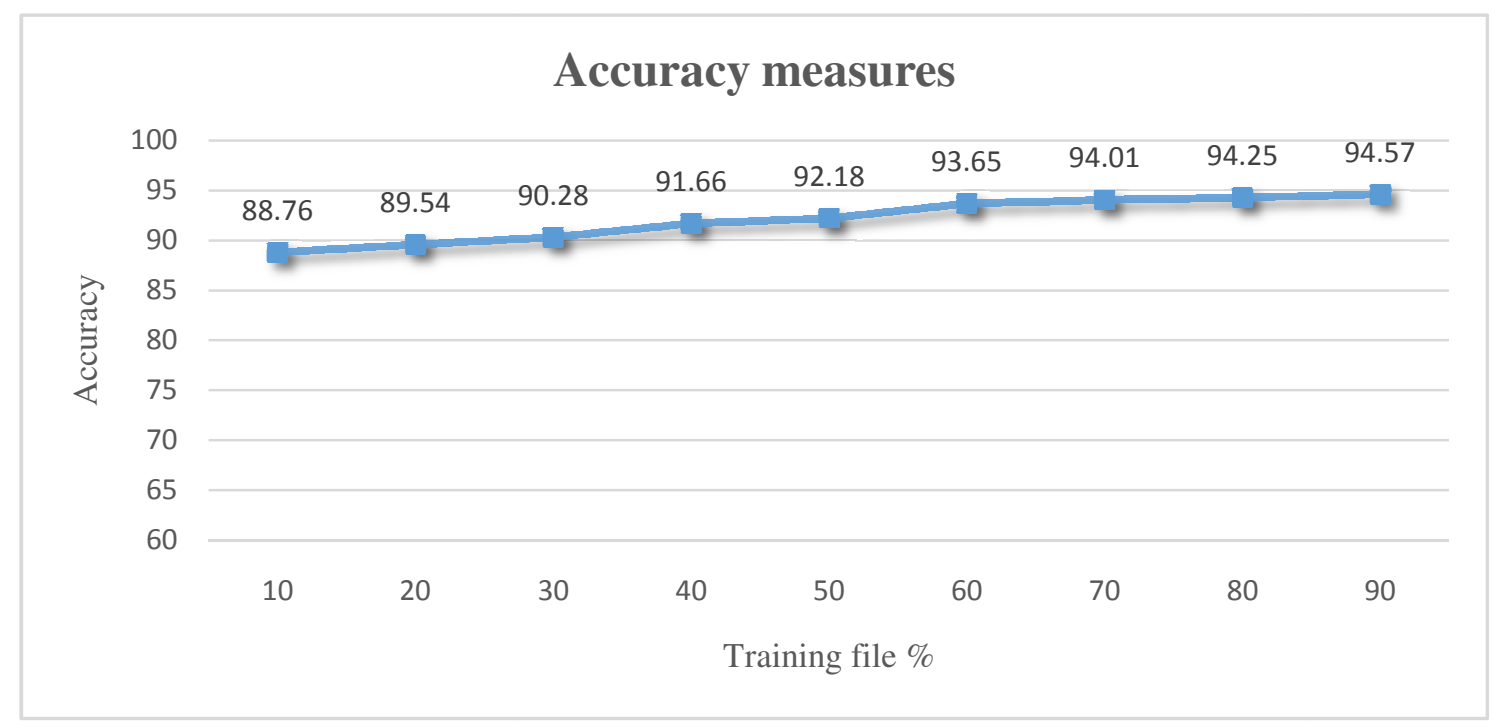

Figure 12. Graph for Accuracy with training files

\section{ApPlication}

Primary face recognition is use for two task 1) verification 2) identification, first: When I was offered an image of the face of a person unknown to the identity claim to determine if the individual is who he / she claim to be. Also known as one to one matching. Second Give an image of the person is unknown and identification of the person by comparing this image with the database of known individual image data. Also known as one to many matching. [11]. 
There are numerous application areas in which face recognition can be exploited for these two purposes, like 1) ATM access control system 2) Public Surveillances 3) General identification 4) Smart card system 5) witness face reconstruction 6) Video indexing and 7) Criminal justice system etc.

\section{Conclusion ANd Future Work}

In this thesis work, we presented a fully automatic face recognition system which works in realtime and which is robust to local illumination changes. In this system we presented novel feature extraction method that is used in face recognition. Every image of face are code into new P-code method which capture both linear directional feature and diagonal directional feature. These two directional features are eight digit code. That is corresponding to all the pixel of face image.

These two directional features capture both straight connectivity and diagonal connectively. We take means value of these two eight digits directional feature. Using means we take both directional feature and also coded into eight digits. So that it is like LBP feature that is proposed in 2002 by co. as compare to LBP feature extraction method LD-BGP feature extraction method is different and capture more useful information about the neighbors to the center pixel.

LD-BGP feature extraction techniques accuracy on face recognition system can be improve by using different preprocessing method in preprocessing phase. Like this accuracy also improve in classification phase using different classifier. This technique is based on LBP and LGS. So that in future new techniques of different graph based can also proposed.

So we think lot of work can possible in LD-BGP feature extraction techniques. But till this techniques work nicely and give good result on face recognition system.

\section{REFERENCES}

[1] Eimad E.A. Abusham and Housam K. Bashir, (2011) "Face Recognition Using Local Graph Structure (LGS)", Springer-journal-Verlag Berlin Heidelberg Human-Computer Interaction, Part II, HCII 2011, LNCS 6762, page no. 169-175.

[2] Rabia Jafri and Hamid R. Arabnia,(2009) “A Survey of Face Recognition Techniques”,Journal of Information Processing Systems, Vol.5, No.2.

[3] Mohd Fikri Azli Abdullah, Md Shohel Sayeed, Kalaiarasi Sonai Muthu, Housam Khalifa Bashier, Afizan Azman and Siti Zainab Ibrahim, (2014) "Face recognition with Symmetric Local Graph Structure (SLGS)”, ELSEVIER Expert Systems with Applications journal 41 pp. 6131-6137.

[4] Timo Ahonen, Abdenour Hadid, and Matti Pietik"ainen, (2004) "Face Recognition with Local Binary Patterns”, Springer-Verlag Berlin Heidelberg, journal LNCS 3021, pp 469-481.

[5] Mathew Turk and alex pentland,(1991) “Eigen faces for recognition”, vision modeling Group the media laboratory Massachusetts Institute of technology journal of cognitive neuroscience Vol. 3, No. 1.

[6] Suman Kumar Bhattacharyya, Kumar Rahul,(2013) "FACE RECOGNITION BY LINEAR DISCRIMINANT ANALYSIS” International Journal of Communication Network Security, ISSN: 2231 - 1882, Vol. 2, Issue-2,

[7] sadhna sharma,(2013) "Template Matching Approach for Face Recognition System" International Journal of Signal Processing Systems Vol. 1, No. 2. 
International Journal in Foundations of Computer Science \& Technology (IJFCST) Vol.6, No.2, March 2016

[8] M.S.R.S. Prasad, S.S. Panda, G. Deepthi and V. Anisha, (2011) "Face Recognition Using PCA and Feed Forward Neural Networks", International Journal of Computer Science and Telecommunications Vol. 2, Issue 8.

[9] Anissa Bouzalmat1, Naouar Belghini2, Arsalane Zarghili3 and Jamal Kharroubi, (2011) "Face Detection and Recognition Using Back Propagation Neural Network and Fourier Gabor Filters” Signal \& Image Processing An International Journal (SIPIJ) Vol.2, No.3.

[10] Orl face databse link http://www.cl.cam.ac.uk/research/dtg/attarchive/facedatabase.html Access time 1/08/2015.

[11] Divyarajsinh N. Parmar and Brijesh B. Mehta, (2013) "Face recognition method and applications", Int.J.Computer Technology \& Applications, ISSN: 2229-6093 Vol 4 (1), pp 84-86.

[12] T. Ojala, M. Pietikäinen, and T. Mäenpää, (2002) "Multiresolution gray-scale and rotation invariant texture classification with local binary patterns," IEEE Transactions on Pattern Analysis and Machine Intelligence, Vol. 24, No. 7, pp. 971-987, 2002.

\section{Authors}

Satyendra Rajput is pursuing Mtech (computer science) degree from MANIT Bhopal. His current research interest includes image processing, biometric system, computer Graphics and Computer network.

Dr. Joyti Bharti is renewed faculty in MANIT Bhopal. His Current research interest includes image processing, ComputerVision, computer Graphics, image segment and hiding.

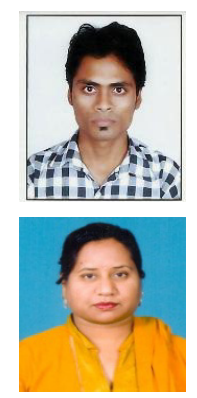

